infants without hypertonia. (Chiriboga CA et al. Neurological correlates of fetal cocaine exposure: Transient hypertonia of infancy and early childhood. Pediatrics December 1995;96:1070-1077). (Reprints: Dr CC Chiriboga, Division of Pediatric Neurology, College of Physicians and Surgeons, Columbia University, 710 West 168 th St, New York, NY 10032).

COMMENT. In our clinic for children with Attention Deficit Disorders at Children's Memorial Hospital, Chicago, I have observed an unusual incidence of a history of fetal cocaine exposure in those placed in foster homes soon after birth. Other complications of cocaine exposure in utero are small head circumference, cerebral infarction or hemorrhage, seizures, and SIDS. Disturbances in corticogenesis have been demonstrated in experiments on laboratory animals (see Progress in Pediatric Neurology I and II, PNB Publishers, 1991, pp452-3, and 1994, pp439-41).

\title{
GLUTEN SENSITIVITY AND NEUROLOGICAL ILLNESS
}

The frequency of IgG and IgA antigliadin antibodies, a measure of cryptic gluten sensitivity, and celiac disease was studied using ELISA in 147 adult patients admitted to the Royal Hallamshire Hospital, Sheffield, UK, for neurologic investigation. Of 53 patients with neurological dysfunction of unknown cause, including 25 with ataxia and 20 with peripheral neuropathy, $30(57 \%)$ had positive antigliadin antibody titers, compared to only $5 \%$ of 94 patients with specific diagnoses, such as stroke, MS, and Parkinsonism, and $12 \%$ of 50 healthy blood donors. In antigliadin-positive patients with ataxia or neuropathy of unknown cause, duodenal biopsies revealed histological evidence of celiac disease in 35\% and non-specific duodenitis in 38\%. Only one had low vitamin B12 levels and the biopsy was normal. Gluten sensitivity was a common finding in this group of adult patients with ataxia and peripheral neuropathy of unknown cause. (Hadjivassiliou $M$ et al. Does cryptic gluten sensitivity play a part in neurological illness? Lancet February 10, 1996;347:369-71). (Respond: Dr M Hadjivassiliou, Department of Clinical Neurology, Royal Hallamshire Hospital, Sheffield S10 2JF, UK).

COMMENT. This investigation underscores the importance of nutrition and diet in some neurological disorders of undetermined etiology. Antigliadin antibody estimation should be considered in the investigation of patients with neurological dysfunction of unknown cause, including those with refractory seizures, and especially if associated with occipital calcifications. Patients with histological evidence of celiac disease are treated with a gluten-free diet. However, those celiac patients with seizure complications and occipital calcifications are not always benefited by diet, and surgical resection of the involved occipital cortex may be required. (see Progress in Pediatric Neurology II, PNB Publishers, 1994, pp71-73).

\section{CRANIAL NERVE DISORDERS}

\section{CONGENITAL FACIAL PALSY}

The association between permanent congenital facial palsy in 61 children and recognized risk factors for traumatic birth was investigated in a retrospective case control study at the Departments of Plastic Surgery, Mount Vernon Hospital, Northwood, Middlesex, and the Hospital for Sick Children, Great Ormond Street, London, UK. The incidence of forceps assisted delivery 